\title{
EDITORIAL
}

\section{How challenging is to manage agitated patients?}

\author{
Daniel A. Cavalcante, ${ }^{1,2}$ iD Ary Gadelha, ${ }^{1,2,3}$ iD Cristiano Noto ${ }^{1,2,3}$ iD \\ ${ }^{1}$ Laboratório Interdisciplinar de Neurociências Clínicas (LiNC), Departamento de Psiquiatria, Universidade Federal de São Paulo (UNIFESP), \\ São Paulo, SP, Brazil. ${ }^{2}$ Grupo de Atenção às Psicoses Iniciais (GAPi), UNIFESP, São Paulo, SP, Brazil. ${ }^{3}$ Programa de Esquizofrenia \\ (PROESQ), UNIFESP, São Paulo, SP, Brazil.
}

Psychomotor agitation is a threatening state of motor restlessness and mental tension that often escalates to aggression and violence. Prompt risk recognition and implementation of adequate protocols may ensure patient and staff safety and resolution of the episode.

Agitated patients are present in most medical care settings. Despite the scarcity of consistent data on the prevalence of episodes of psychomotor agitation in health services, it is known that these phenomena are quite common in medical practice. In the U.S., for example, agitation episodes are estimated to account for 1.7 million visits a year to emergency medical services. ${ }^{1}$ In addition, recent studies conducted in that country and in Europe show that up to $10 \%$ of emergency room visits involve an episode of acute psychomotor agitation..$^{2-4}$ In Brazil, data show that agitation episodes account for about $24 \%$ of psychiatric emergencies. $^{5}$

Although common, psychomotor agitation remains a very challenging situation, associated with a wide range of psychiatric, neurologic, surgical, and internal medical conditions. Although manifestations may be quite similar, adequate management varies greatly, especially regarding medication.

Failure to approach an agitated patient properly can lead to serious consequences, including harm not only to health care professionals and to the patient but also to other patients and/or family members present. Ineffective management may yield tragic results, such as escalation to aggressive/violent behavior or the occurrence of serious adverse effects secondary to inadequate medication use.

The increasing demand for psychiatric care is noticeable, and is partly due to the reduction of stigma surrounding psychiatric diseases and treatments. Improved management of psychomotor agitation is one of the factors that contribute to the reduction of this stigma, as it leads to a lower occurrence of side effects and a lower frequency of mechanical restraint use in hospitalized patients. It is also noticeable that this increasing demand in Brazil is making psychiatric emergency facilities even more crowded, and consequently harder to manage.

It is imperative, therefore, that strategies be developed to reduce flaws in the management of such frameworks. The Brazilian Journal of Psychiatry contributes to this

Correspondence: Daniel A Cavalcante

E-mail: daniel_cavalcante10@ hotmail.com debate by presenting the Brazilian guidelines for the management of psychomotor agitation, ${ }^{6,7}$ a commendable initiative by the Brazilian Psychiatric Association (Associação Brasileira de Psiquiatria [ABP]) to address a major gap in the literature. In recent years, few guidelines have been developed specifically taking into account the reality of low- and middle-income countries (LAMIC), where some of the medications commonly recommended in international guidelines are not available.

It is also noteworthy that this publication plays an important role as it brings to the discussion an issue of central importance not only to psychiatrists but to clinicians in general. We encourage not only $A B P$, but also other psychiatric associations in LAMICs, to expand the initiative of developing clinical guidelines for other sensitive areas of psychiatric care, adapted to the local reality, combining scientific evidence and practical concerns.

\section{Disclosure}

DAC has received fees as a speaker for Torrent. AG has participated in advisory boards for Janssen and Daiichi Sankyo; and has received fees as a speaker for DaichiiSankyo, Ache, Janssen and Torrent. CN has participated in advisory boards for Janssen; and has received fees as a speaker for Ache, Biolab, Daiichi Sankyo, Janssen and Torrent.

\section{References}

1 Sachs GS. A review of agitation in mental illness: burden of illness and underlying pathology. J Clin Psychiatry. 2006;67 Suppl 10: 5-12.

2 San L, Marksteiner J, Zwanzger P, Figuero MA, Romero FT, Kyropoulos $\mathrm{G}$, et al. State of acute agitation at psychiatric emergencies in Europe: the STAGE study. Clin Pract Epidemiol Ment Health. 2016;12:75-86.

3 Pascual JC, Madre M, Puigdemont D, Oller S, Corripio I, Díaz A, et al. A naturalistic study: 100 consecutive episodes of acute agitation in a psychiatric emergency department. Actas Esp Psiquiatr. 2006;34: 239-44.

4 Pajonk FG, Schmitt P, Biedler A, Richter JC, Meyer W, Luiz T, et al. Psychiatric emergencies in prehospital emergency medical systems: a prospective comparison of two urban settings. Gen Hosp Psychiatry. 2008;30:360-6.

5 Santos ME, do Amor JA, Del-Ben CM, Zuardi AW. Psychiatric emergency service in a university general hospital: a prospective study. Rev Saude Publica. 2000;34:468-74.

How to cite this article: Cavalcante DA, Gadelha A, Noto C. How challenging is to manage agitated patients? Braz J Psychiatry. 2019;41:277-278. http://dx.doi.org/10.1590/1516-4446-2019-4105 
6 Baldaçara L, Diaz AP, Leite V, Pereira LA, dos Santos RM, Gomes Júnior VP, et al. Brazilian guidelines for the management of psychomotor agitation. Part 2. Pharmacological approach. Braz J Psychiatry. 2019;41:324-35.
7 Baldaçara L, Ismael F, Leite V, Pereira LA, dos Santos RM, Gomes Júnior VP, et al. Brazilian guidelines for the management of psychomotor agitation. Part 1. Non-pharmacological approach. Braz J Psychiatry. 2019;41:153-67. 